\title{
Design of a Linear Variable Differential Transformer With High Rejection to External Interfering Magnetic Field
}

\author{
M. Martino ${ }^{1,2}$, A. Danisi ${ }^{1,3}$, R. Losito ${ }^{1}$, A. Masi ${ }^{1}$, and G. Spiezia ${ }^{1}$ \\ ${ }^{1}$ CERN, EN/STI Group, Geneva 1211, Switzerland \\ ${ }^{2}$ Electrical Engineering Department, University of Naples "Federico II," Naples 80138, Italy \\ ${ }^{3}$ Electronic and Telecommunications Engineering Department, University of Naples "Federico II," Naples 80138, Italy
}

\begin{abstract}
The sensitivity of linear variable differential transformer (LVDT) position sensors to external slowly varying magnetic fields represents a critical issue when these sensors are installed close to high-current cables or electrical motors with significant fringe fields. The resulting position error can reach several hundreds of micrometers against a specified uncertainty normally below a few micrometers. In this paper, the design of a LVDT position sensor with high rejection to external constant or slowly varying magnetic fields is addressed by exploiting the finite element method (FEM) simulator FLUX. A shield, isolated from the sensor's magnetic circuit, has been considered to reduce the effect of magnetic fields on the secondary voltages of the LVDT. In addition, a dc current is used in order to polarize the magnetic circuit to reduce the sensitivity of the sensor to external interferences.
\end{abstract}

Index Terms-Linear Variable Differential Transformer (LVDT), magnetic interference, magnetic shielding.

\section{INTRODUCTION}

$\mathbf{T}$ HE linear variable differential transformer (LVDT) is one of the position sensors most used in harsh environments, such as industrial plants, nuclear plants, and particle accelerators, thanks to contactless sensing, good linearity, virtually infinite resolution, low temperature sensitivity, robustness, and easy-to-implement radiation hardness [1]. As an example, LVDT sensors have been selected for the LHC (Large Hadron Collider) collimators, where integrated doses of up to a few MGray per year are expected [2].

The resolution and the accuracy of such a sensor are mainly determined by the conditioning electronics and the correction algorithm used [3]. Digital conditioning approaches [4], [5], together with a ratiometric reading technique [1], ensure high linearity and reading accuracy, even in conditions of low signal-tonoise ratio. For instance, in the LHC collimators application, a target uncertainty of $20 \mu \mathrm{m}$ on the position measurement has been achieved [6].

In applications where such a high accuracy is required, dc and slowly varying magnetic fields may drastically interfere with the LVDT, causing measurement errors. In the LHC transfer lines collimators, the LVDT sensors experience a maximum external flux density of $1 \mathrm{mT}$ due to the high-current cables supplying the transfer line magnets and passing a few tenths of a meter away from them. During the magnets powering cycle, the LVDT reading is affected by a measurement error of some hundreds of micrometers, much greater than the required reading uncertainty of $20 \mu \mathrm{m} \mathrm{[7].}$

In this paper, the design of an LVDT position sensor with high rejection to external constant or slowly varying magnetic fields is addressed by exploiting a finite element method (FEM) simulator [8]. The goal is to reduce the expected external magnetic field of $800 \mathrm{~A} / \mathrm{m}$ ( $1 \mathrm{mT}$ magnetic flux density in air) to about $30 \mathrm{~A} / \mathrm{m}$, which will give a measurement error less than $20 \mu \mathrm{m}$, as it will be shown in detail in the experimental results. The in-

Manuscript received June 21, 2009; revised September 03, 2009; accepted September 14, 2009. Current version published January 20, 2010. Corresponding author: M. Martino (e-mail: michele.martino@cern.ch).

Digital Object Identifier 10.1109/TMAG.2009.2033341

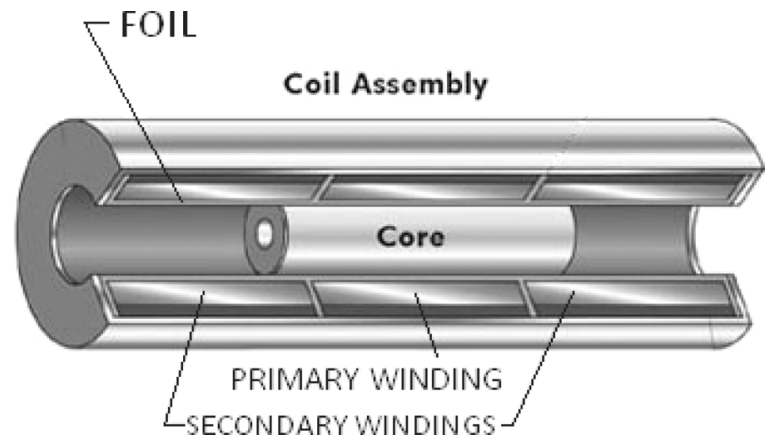

Fig. 1. LVDT magnetic circuit and working principle (courtesy of Macrosensors).

novative solution is obtained with the combined effects of an external shield, isolated from the sensor magnetic circuit, and a dc magnetic polarization of the LVDT magnetic circuit.

In Section II, after a brief recall of the LVDT working principle, the problem of the interaction with an external magnetic field is introduced. In Section III, after introducing the LVDT model used for simulations, the new proposed design aimed to increase the rejection to the interfering magnetic field is presented and discussed. In Section IV, the simulation results are shown.

\section{THE PROBLEM}

The LVDT, an inductive positioning sensor, is basically a transformer with one excitation, or primary, coil fed by a sinusoidal voltage source and two reading, or secondary, coils wound on a support, referred to as foil from now on (Fig. 1). The core of the transformer is a movable part made of soft magnetic material (such as nickel-iron alloy), which is bound to the element whose position is to be measured (Fig. 1). When the core moves toward a coil, the amplitude of the magnetic flux, produced by the primary winding and linked to that coil, increases. Accordingly, the voltage across it increases, too, while the amplitude of the voltage across the other coil decreases. The difference between the secondary coil voltages allows evaluating the core position. 
To the best of our knowledge, there are no scientific papers addressing specifically the influence of an external magnetic field on an LVDT. Datasheets of commercial LVDTs sometimes warn the user about that, but no quantitative information is given. A detailed description of this phenomenon outgoes the scope of this work. The basis of such a phenomenon can be understood by referring to the working principle of fluxgate magnetometers and direct current current transformers (DCCTs) [9]-[11].

Let us consider an equivalent model of the overall primary and secondary circuits neglecting the hysteresis of the minor loops. It is possible to show that the amplitude $A_{1,2}^{f_{0}}$ of the first harmonics of the secondary voltages can be expressed (truncating its series expansion at the first order) by the following approximated equation [12]:

$$
A_{1,2}^{f_{0}} \approx S_{\mathrm{sec}} 2 \pi f_{0} H_{\mathrm{ac}}\left|\mu_{d_{1,2}}^{\mathrm{eq}}\left(H_{\mathrm{dc}}, x\right)\right|
$$

where $\mu_{d_{1,2}}^{\mathrm{eq}}$ is the equivalent differential permeability of the secondary circuits that takes into account the different magnetic materials and the relative volumes occupied by them. $x$ is the core position, $S_{\mathrm{sec}}$ is the area of both the secondary coils, $H_{\mathrm{ac}}$ and $H_{\mathrm{dc}}$ are the magnetic fields produced respectively by the primary excitation at frequency $f_{0}$ and the external interfering field. Because of the nonlinearity of the B-H curve of each magnetic material, a variation in the external field results in a variation in the amplitudes of the secondary voltages, resulting in a position measurement error.

In complete analogy to a simple fluxgate [9], the outmost interfering component of the external field is the longitudinal one, as it will be discussed in Section IV. Therefore, the design is mainly focused on the rejection of this component.

\section{PROPOSAL}

For the purpose of this paper, the LVDT design is not referred to a particular commercial sensor, therefore typical LVDT parameters such as sensitivity and primary voltage excitation are taken into account. The geometrical dimensions are purely indicative. A maximum interfering magnetic field of $800 \mathrm{~A} / \mathrm{m}$ ( $1 \mathrm{mT}$ of magnetic flux density in air) has been considered. This represents a reasonable value, extrapolated from examples of critical installations [7], [13]. Anyway, the design guidelines discussed here can be easily applied to stronger interference cases. As a proposed target, the effect of the external field has to be reduced such that the position sensor uncertainty must be contained within $20 \mu \mathrm{m}$.

The rejection of the longitudinal interfering magnetic field on the LVDT readings is obtained by means of a proper designed shield and enhanced by applying a dc polarization of the magnetic media.

\section{A. LVDT Model}

The primary coil is wound along the entire length of the sensor under study (about $110 \mathrm{~mm}$ ), whereas the secondary windings are wound over the primary, one on each side of the bobbin. The ferromagnetic core is a hollow cylinder whose length matches the one of the secondary windings (about $50 \mathrm{~mm}$ ). The core is made up of Alloy 48, a very high relative permeability material, commonly used for these purposes
[14], [15]. The foil is made up of S235 steel, which exhibits ferromagnetic behavior and relatively high permeability. The soft magnetic materials have been chosen to assure a working voltage swing greater than $1 \mathrm{~V}$. In addition, the choice takes into account the fact that the LVDT materials themselves may worsen the interference effect. The model presents insulator layers and washers between and over the primary and secondary coils that are treated as nonmagnetic regions in the simulations. The structure is enclosed in a cylindrical case with two end-caps, made up of S235 steel.

\section{B. DC Polarization}

The sensitivity of the amplitude of the secondary voltages to the external field depends clearly on the local slope of $\mu_{d}^{\mathrm{eq}}$ around the generic point $H_{\mathrm{dc}}^{0}$, as shown by evaluating the difference quotient

$$
\frac{\Delta A_{1,2}^{f_{0}}}{\Delta H_{\mathrm{dc}}} \approx S_{\mathrm{sec}} 2 \pi f_{0} H_{\mathrm{ac}} \frac{\Delta\left|\mu_{d_{1,2}}^{\mathrm{eq}}\right|}{\Delta H_{\mathrm{dc}}}\left(H_{\mathrm{dc}}^{0}, x\right) .
$$

The field $H_{\mathrm{dc}}^{0}$ is zero in the nominal operation of the LVDT, so the sensitivity of the sensor to the external field depends on the slope of the differential permeability of the different materials around the zero.

Actually, the $H_{\mathrm{dc}}$ field can be equivalently produced applying a dc component in the primary circuit. In this case, analyzing the typical shape of the B-H curve of the used soft magnetic materials [14], it is possible to choose a biasing field $H_{\mathrm{dc}}^{0}$ characterized by a reduced sensitivity to the $\Delta H_{\mathrm{dc}}$ produced by the external field.

Because of the demagnetization effect on the core of the LVDT, a relationship between the applied field produced by the primary current and the resulting fields $H_{\mathrm{ac}}$ and $H_{\mathrm{dc}}$ can be established only by means of simulations. Finally, such biasing will affect the linearity of the sensor [16].

\section{Shield Design}

Normally, commercial LVDTs present a cylindrical shield integrated in the sensor magnetic circuit.

An innovative aspect of the presented solution consists in separating the magnetic circuit of the coaxial cylindrical shield from that of the sensor. By doing so, the design of the shield can be optimized for the rejection without concerning any magnetic sensor constraint.

Dimensions, materials, and distances between the different shielding layers can be adjusted to achieve a given shielding efficiency, depending on the maximum acceptable position drift. Preliminary simulations have shown that a 1-mT transversal flux density produces a nonuniform distribution along the core, ranging from 3 to $19 \mu \mathrm{T}$, whereas a $1 \mathrm{mT}$ longitudinal flux density produces a nonuniform distribution along the core, ranging from 8 to $130 \mathrm{mT}$. Thus, the effect of a transversal field is negligible with respect to the longitudinal one. A set of simulations has been carried out to evaluate the effect of an interfering longitudinal magnetic field, ranging from 0 to $800 \mathrm{~A} / \mathrm{m}$, on the measured position. It showed that the maximum drift on the measured position is about $1 \mathrm{~mm}$, as well as that the magnetic field has to be reduced to about $35 \mathrm{~A} / \mathrm{m}$ in order to have a drift not greater than $20 \mu \mathrm{m}$. Therefore, the shield has to show an attenuation factor of about 23 . 


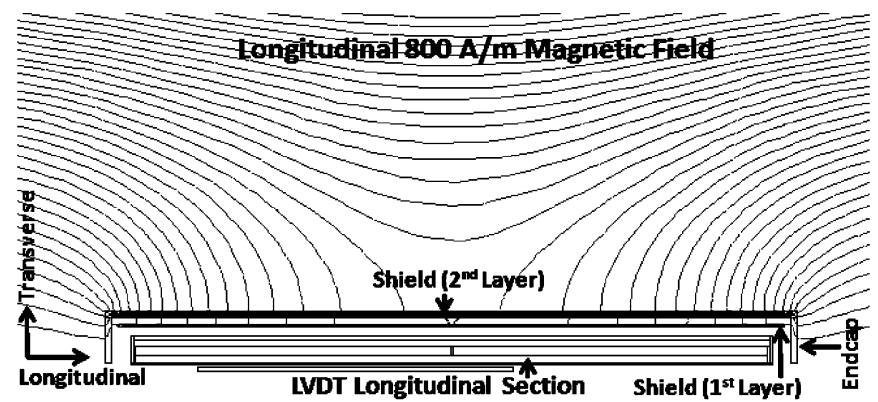

Fig. 2. Flux density distribution in case of an external longitudinal magnetic field impinging on the LVDT shield. Note that only half of the LVDT longitudinal section is displayed, being symmetric around the axis.

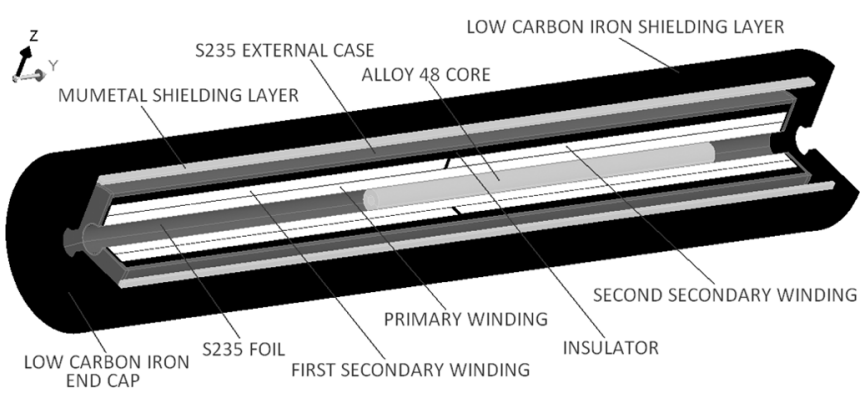

Fig. 3. LVDT model prototype and shield case. Half of the displayed longitudinal section has been used for 2D simulations. The iron layer exhibits a thin air gap between the cylindrical part and the caps in order to take into account the actual physical discontinuity between the two pieces.

In a scenario in which a longitudinal interfering field of $800 \mathrm{~A} / \mathrm{m}$ is considered, the cylindrical shield is interested by a field with both longitudinal and transversal components not uniformly distributed along its length (Fig. 2).

Since such a case cannot be handled by means of an analytical approach [17], a simulation plan with FEM software turned out to be necessary in order to design the shield, having as a basis the following guidelines: 1) use of high magnetic permeability materials to reduce the interfering fields; 2) consideration of a multilayer shield to prevent the saturation of the inner layer; 3) optimization of the air gap between the layers and their thicknesses not to oversize the shield with respect to the sensor dimensions.

Accordingly, a single-layer shield with a high-permeability material, such as Mumetal [18], has been discarded since a decrease of about $50 \%$ of the permeability (from 120000 to 60000 ) has been observed, meaning a significant saturation. For this reason, the shield has been endowed with an external layer (Fig. 3). The distance between the two layers is $1 \mathrm{~mm}$, and it assures that the Mumetal layer does not saturate and provides an appropriate shunting for the flux. The external layer is a low-carbon iron cylindrical foil [18] endowed with two end-caps that, in particular, assure a very effective binding of the longitudinal flux lines, avoiding a direct impact on the sensor's magnetic circuit, as shown in Fig. 2. Owing to that, additional Mumetal end-caps turned out to be useless.

A simulation scan showed that $1 \mathrm{~mm}$ of low-carbon iron and $0.4 \mathrm{~mm}$ of Mumetal assure both the saturation prevention and the required shield efficiency. In fact, with an external magnetic field of about $30 \mathrm{~A} / \mathrm{m}$, the drift of the measured position of the

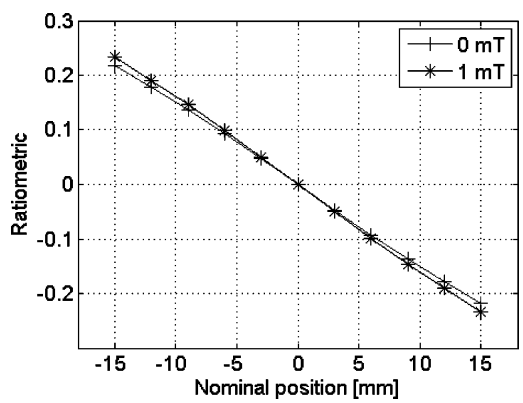

Fig. 4. Sensor characteristic with and without a longitudinal magnetic field of $800 \mathrm{~A} / \mathrm{m}$.

TABLE I

MESH PROPERTIES

\begin{tabular}{ll}
\hline \hline \multicolumn{1}{c}{ Indicator } & \multicolumn{1}{c}{ Description } \\
\hline Mesh Type & 2D Triangular Mesh, Nonuniform \\
Mesh Order & $2^{\text {nd }}$ order \\
Number of Mesh Elements & 10628 \\
Number of Poor Quality Elements & $3.5 \%$ \\
Min - Max Mesh Element Size & $0.05 \mathrm{~mm}$ (Foil) $-20 \mathrm{~mm}$ (Air $\infty)$ \\
Average Mesh Density & 3 elements/thickness \\
\hline
\end{tabular}

The table explains the mesh properties. The choice of a nonuniform $2^{\text {nd }}$ order mesh assures a faster elaboration and a precise solution.

LVDT is contained within $20 \mu \mathrm{m}$, which has been considered as the design goal.

\section{The Simulation Validation}

\section{A. FEM Modeling}

The FEM simulator FLUX from CEDRAT Technologies has been used, as it is particularly suited for solving electromagnetic problems involving moveable parts, such as sensors, motors, and actuators [8]. In addition, it allows a deep investigation of flux distributions, end effects, nonlinearity, and nonuniformity of field strength along the different magnetic elements [19], [20]. For ferromagnetic materials, FLUX works with nonlinear B-H curves, but it neglects the magnetic hysteresis effects [8].

The model has been prepared in $2 \mathrm{D}$ environment, taking into account the fact that the LVDT sensor has cylindrical symmetry, so only half of the longitudinal section of the sensor has been modeled (Fig. 2). Table I summarizes the model mesh features.

The primary coil is fed by a voltage generator providing a $3.5-\mathrm{V}$ sine waveform at $2000 \mathrm{~Hz}$. The amplitude of the first harmonics of the induced voltages on the two secondary coils has been evaluated using a fast Fourier transform (FFT) algorithm in order to calculate the ratiometric value as a function of the position, ranging from -15 to $15 \mathrm{~mm}$, corresponding to $60 \%$ of the overall core position range of the sensor.

\section{B. Simulation Results}

The following simulation results take into account only the interference of the longitudinal magnetic field, which is the dominating interfering component.

Fig. 4 shows the characteristic of the sensor, representing the calibration curve, and the effects on it of an $800-\mathrm{A} / \mathrm{m}$ external magnetic field. The position value, in presence of the external 


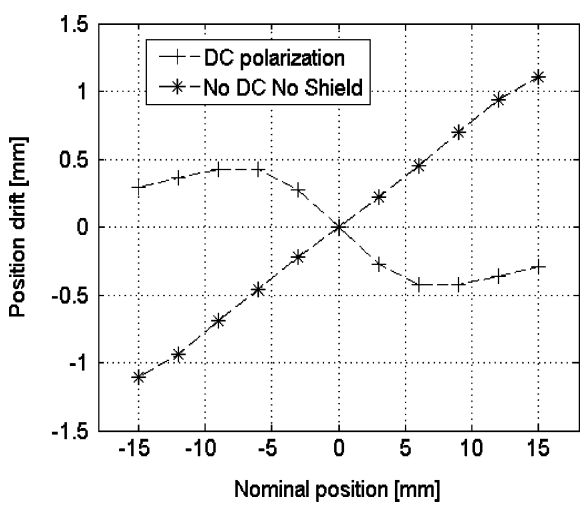

Fig. 5. Comparison between the position drift of the LVDT model without (+) and with $(*)$ dc polarization.

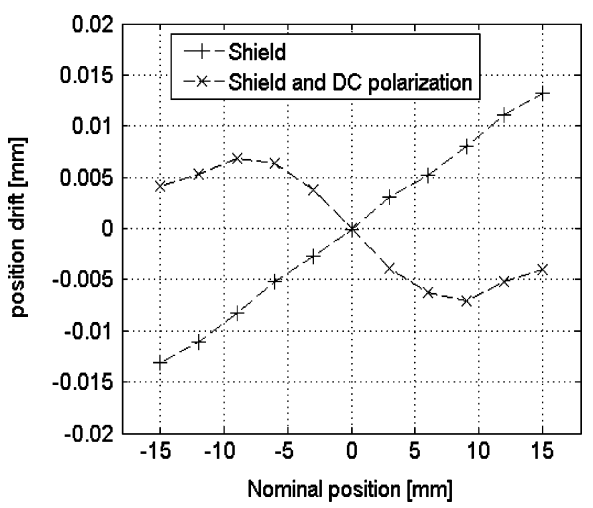

Fig. 6. Position drift with the shield case (+) and with its combined effect with dc polarization $(*)$

field, has been evaluated on the basis of the corresponding ratiometric value by means of a linear interpolation of the calibration curve. The position drift increases according to the nominal position, achieving a maximum value of about $1 \mathrm{~mm}$ (Fig. 5).

As mentioned, a dc magnetic bias can be used to reduce the effect for those cases where a shield is not sufficient or cannot be fit for mechanical reasons. To this purpose, a dc voltage of $10 \mathrm{~V}$ (corresponding to $160 \mathrm{~mA} \mathrm{dc}$ current) has been added to the primary sine-wave input. Once the calibration curve of the sensor in presence of the dc effect is evaluated, the maximum position drift due to an external interference of $800 \mathrm{~A} / \mathrm{m}$ results to be reduced by a factor 2 (Fig. 5). The inversion of the position drift sign is due to the dc polarization that affects the characteristic curve of the sensor.

However, as a main solution strategy, the external magnetic interference can be reduced by means of the shield case described in the previous section. Fig. 6 shows that the shield reduces the position drift to about $12 \mu \mathrm{m}$ without deeply affecting the sensor linearity.

Finally, both the shield case and the $10-\mathrm{V}$ dc polarization were applied to the LVDT (Fig. 6). It can be stated that the benefits of both the solutions are kept since the position drift is still reduced by a factor 2 with respect to the case in which the only shield was considered (Fig. 6).

\section{CONCLUSION AND OUTLOOK}

The interference of a dc or slowly varying magnetic field with an LVDT has been investigated. A strategy to improve the re- jection to external longitudinal interfering field has been proposed and validated by means of a FEM simulation approach. In particular, a proper shielding design, isolated from the LVDT magnetic circuit and enhanced with primary dc polarization, has been discussed. In some cases, these countermeasures can even be adopted on commercial and already-designed LVDTs in order to improve rejection performances. Future works concern the prototype realization and the experimental validations as well as a detailed analytical LVDT model, able to foresee and quantify the interference effects.

\section{ACKNOWLEDGMENT}

Authors would like to thank Prof. V. G. Vaccaro, Prof. F. Cennamo, and Prof. P. Arpaia for their useful discussions and suggestions. They would also like to thank Dr. D. Taghezout from Applied Magnetics for his support on the simulations.

\section{REFERENCES}

[1] K. Ara, "A differential transformer with temperature- and excitationindependent output," IEEE Trans. Instrum. Meas., vol. IM-21, no. 3, pp. 249-255, Aug. 1972.

[2] A. Masi and R. Losito, "LHC collimators low level control system," IEEE Trans. Nucl. Sci., vol. 55, no. 1, pp. 333-340, Feb. 2008.

[3] R. S. Weissbach, D. R. Loker, and R. M. Ford, "Test and comparison of LVDT signal conditioner performance," in Proc. 17th IEEE Instrum. Meas. Technol. Conf., 2000, pp. 1143-1146.

[4] S. K. Mishra and G. Panda, "A novel method for designing LVDT and its comparison with conventional design," in Proc. IEEE Sensors Appl. Symp., Houston, TX, 2006, pp. 129-134.

[5] D. Crescini, A. Flammini, D. Marioli, and A. Taroni, "Application of an FFT-based algorithm to signal processing of LVDT position sensors," IEEE Trans. Instrum. Meas., vol. 47, no. 5, pp. 1119-1123, Oct. 1998.

[6] A. Masi, A. Brielmann, R. Losito, and M. Martino, "LVDT conditioning on the LHC collimators," IEEE Trans. Nucl. Sci., vol. 55, no. 1, pp. 67-75, Feb. 2008.

[7] A. Danisi, "Simulation of dc interfering magnetic field effects on the LHC collimators' LVDT positioning sensors," M.S. thesis, Dept. Electron. Eng., Univ. "Federico II", Naples, IT, 2009, EDMS Id 1016998 [Online]. Available: https://edms.cern.ch/

[8] “Flux 9 2D/3D Applications User's Guide, Vol. 2," CEDRAT, Meylan, France, 2006.

[9] S. V. Marshall, "An analytic model for the fluxgate magnetometer," IEEE Trans. Magn., vol. MAG-3, no. 3, pp. 459-463, Sep. 1967.

[10] I. Sasada, "Orthogonal fluxgate mechanism operated with dc biased excitation," J. App. Phys., vol. 91, no. 10, pp. 7789-7791, May 2002.

[11] P. Odier, "DCCT technology review," in Proc. CARE-Conf-2004-023HHH, Lyon, France, Dec. 2004.

[12] M. Martino, "Characterization of magnetic interference on LVDT positioning sensor," Mar. 2009, unpublished.

[13] S. Nagaitsev, C. Gattuso, S. Pruss, and J. Wolk, "Experience with magnetic shielding of a large scale accelerator," in Proc. 2001 Particle Accelerator Conf., vol. 5, pp. 3371-3373.

[14] R. Bozorth, "Iron-Nickel Alloys," in Ferromagnetism, 3rd ed. New York: Van Nostrand, 1951, pp. 130-131.

[15] K. Hoselitz, "Magnetically soft materials," in Ferromagnetic Properties of Metals and Alloys. Oxford, U.K.: Clarendon, Oxford Univ. Press, 1952, pp. 238-239.

[16] S. Wu, S. Mo, and B. Wu, "An LVDT-based self-actuating displacement transducer," Sensors Actuators A. vol. 141, no. 2, pp. 558-564, Feb. 2008 [Online]. Available: Available: www.sciencedirect.com

[17] Designers Handbook: The When, Why and How of Magnetic Shielding. Blairsville, PA: Westinghouse, 1966.

[18] “Metals Handbook vol. 1,” 10th ed. ASM International, 1990.

[19] J. K. Sykulski, E. Sykulska, and S. T. Hughes, "Application of finite element modelling in LVDT design," COMPEL, vol. 11, no. 1, pp. 73-76, 1992.

[20] M. Repetto and J. Simkin, "Engineering analysis for design optimization of differential transformers," IEEE Comput.-Aided Eng. J., vol. 5, no. 2, pp. 51-53, Apr. 1988. 